\title{
The ensemble-averaged characteristics of the bubble system during cavitation in the separator
}

\author{
Anna Kapranova ${ }^{1}$, Anton Lebedev ${ }^{1}$, Sergey Neklyudov² and Alexander Melzer ${ }^{2}$ \\ ${ }^{1}$ Yaroslavl State Technical University, Yaroslavl, Russian Federation \\ ${ }^{2}$ JSC “Regulyator”, Yaroslavl, Russian Federation
}

\begin{abstract}
The object of research is the process of formation of vapor-gas bubbles at an early stage of hydrodynamic cavitation during throttling of the liquid in the axial valve separator. Designing a separator under conditions of decreasing bubble formation intensity is an urgent problem. Based on the energy approach, a stochastic model of the formation of an energetically closed system of bubbles in the separator according to the Gibbs ensemble is proposed. Analytical dependences are obtained for the density function and the bubble geometric characteristics averaged over the ensemble, depending on the design parameters, operating parameters, and physic-mechanical properties of the working medium, including the ratio of the gas and vapor contents in the inner cavity of the bubble. The expressions for the indicated distribution function over the size of the bubbles and for the ensemble-averaged characteristics of the system of bubbles formed (diameter and surface area) can be used in the formation of an engineering methodology for calculating control equipment.
\end{abstract}

\section{Introduction}

The phenomenon of bubble cavitation is of particular interest for multiphase flow processes in axial valve separators. An active study of this phenomenon is associated with the need to design equipment with an increased durability due to erosion of the inner surfaces of the valves with a guaranteed safe limit of noise effects and vibrations of structural elements. Studying the mechanism of the appearance of vapor-gas bubbles in the indicated flows at the stage of initial hydrodynamic cavitation before their growth and collapse helps to further predict the behavior of the bubble system at the developed stage of cavitation. The problem of describing this mechanism was raised in classical works [1-3].

Evaluation of the size of the bubble in the valve separator depends on many factors, including the physical state of the liquid medium, a set of design and operational parameters of the device. The identification of valve operating modes with a reduced intensity of cavitation bubble formation is one of the most important tasks in the design of pipeline valves. However, the development of the theoretical foundations of the bubble cavitation process at its early stage remains an urgent problem [4, 5]. Known deterministic approaches to the description of the mechanism of bubble cavitation associated with solving systems of equations of mechanics of heterogeneous media have recently become dependent on the implementation of numerical simulation methods [6-8].
Stochastic approaches [9] in solving this problem, as a rule, are based on kinetic equations obtained by analogy with equations from the theory of Brownian particle motion for the nucleation process $[10,11]$ in media with different degrees of viscosity taking into account surface effects. The variety of problems in modeling the nucleation process is determined by a homogeneous [10,11] (or modified homogeneous [12]) and heterogeneous [13] mechanisms. Moreover, in the latter case, features of the formation of vapor bubbles on the boundary surfaces of various nature are revealed [14], including in electrochemical systems [15]. In addition, in the simulation of bubble cavitation, the Euler-Lagrange approach is used, when it is proposed to use various variables to describe the behavior of the phases of the hydrodynamic system taking into account the influence of interphase transfers [16], including when combined with the process of liquid degassing [17]. The density functions used in the models for bubble cavitation are often semi-empirical in nature, and the desired solutions are numerically represented.

The well-known stochastic approach presented in [18] allows one to describe random processes from the standpoint of both the energy closure of the system by the Gibbs ensemble and the energy openness of the system in the presence of macroscale fluctuations of its states. In particular, the application of the energy method [19] for the latter case was tested by the authors on the example of modeling the motion of rarefied particles during their mixing $[19,20]$. The aim of this work is to 
obtain analytical dependences for the ensemble-averaged characteristics of the system of generated bubbles in the axial valve separator, taking into account the main parameters of its design [21] and the safe operation mode. The practical application of the results relates to the design of regulatory equipment in conditions of decreasing intensity of bubble cavitation. To achieve this goal, it is necessary to identify the most significant factors affecting the appearance of bubbles in the axial valve separator, set a sufficient set of input parameters for the stochastic model based on the approach [18], and form a differential function for the distribution of bubbles by their size based on the works of the authors [22-24].

\section{Methods}

Suppose that when a fluid moves with a density $\rho_{L}$ in the axial valve separator [21], a system of bubbles is formed at an early stage of hydrodynamic cavitation and is a macro-system of spherical cavities of radius $r$ filled with steam and gas in the ratio $\alpha_{p}: \alpha_{g}$ under conditions of energy closure by the Gibbs ensemble. Taking into account the accepted approximations from [24] on the importance of Hamilton coordinates and momenta in the direction of the bubble's center of mass movement, the specific values of the bubble radius $\lambda=r / r_{c}$ and the velocity of its center of mass $\mu=v / v_{L}$ for the phase volume element $d u=r_{c} \nu_{L} d \lambda d \mu$ are taken as phase variables, where $r_{c}, v_{L}$ are the characteristic values for the radius of the spherical bubble and fluid flow rates in the valve separator.

According to the stochastic approach [18], the construction of a stationary solution of the FokkerPlanck kinetic equation in the energy representation for the equilibrium bubble distribution function $\varphi(\lambda, \mu)=A \exp \left[-E(\lambda, \mu) / E_{0}\right]$ is performed depending on the indicated phase variables [25]. It is indicated here: $A=N^{-1} \int_{u} \exp \left[-E(\lambda, \mu) / E_{0}\right] d u \quad$ is normalization parameter; $E(\lambda, \mu)$ is the energy of the bubble during its stochastic movement; $E_{0}$ is the energy parameter equal to the stationary value of the bubble energy at the time of its stochastization.

Then the differential distribution function of the bubbles by their specific sizes and the number of bubbles in the phase volume element $d u$ are determined by the expressions

$$
\begin{gathered}
\varphi_{1}(\lambda)=N^{-1} d N / d \lambda, \\
d N=A \exp \left[-E(\lambda, \mu) / E_{0}\right] d u .
\end{gathered}
$$

In accordance with the method of constructing the bubble energy during its stochastic motion $E(\lambda, \mu)$, proposed in [22], we have

$$
E(\lambda, \mu)=\left(v_{L} \mu\right)^{2} q_{1}(\lambda)+q_{2}(\lambda),
$$

where the dependences $q_{1}(\lambda), q_{2}(\lambda)$ on the specific radius of the bubble are indicated as follows

$$
\begin{gathered}
q_{1}(\lambda)=\left[k_{1}\left(r_{c} \lambda\right)^{4}+k_{2}\right] /\left(r_{c} \lambda\right), \\
q_{2}(\lambda)=k_{3} M^{2} /\left(r_{c} \lambda\right)^{5}+\left(k_{4}+k_{5} r_{c} \lambda\right)\left(r_{c} \lambda\right)^{2} .
\end{gathered}
$$

The coefficients $k_{j}, j=\overline{1,5}$ in (4), (5) are given by the expressions:

$k_{1}=2 \pi\left(\alpha_{g} \rho_{g}+\alpha_{s} \rho_{s}\right) / 3 ; k_{2}=k_{\zeta} \rho_{L} \zeta_{12} / 4$;

$k_{3}=5 k_{1} / 8 ; k_{4}=4 \pi \sigma ; k_{5}=8 \pi p_{s s} / 3$ where $\rho_{g}, \rho_{s}$ are the densities of gas and vapor; $\zeta_{12}=\left(5,04 \cdot 10^{4} \omega_{y} / K_{v y}\right)^{2}$ is the value of the coefficient of hydraulic resistance during fluid movement in the transition region for the next limit of change of the Reynolds criterion $10<\operatorname{Re}<10^{4}$ for the conditional cross-sectional area $\omega_{y}\left(\mathrm{~m}^{2}\right)$ for the conditional valve capacity $K_{v y}\left(\mathrm{~m}^{3} / \mathrm{h}\right)$; $k_{\zeta}$ is coefficient of proportionality; $p_{\text {ss }}$ is coefficient of surface tension of the liquid; $\sigma$ - saturated vapor pressure. Note that the nominal diameter $D_{y}=\left(4 \omega_{y} / \pi\right)^{1 / 2}(\mathrm{~m})$ is related to the nominal section area $\omega_{y}\left(\mathrm{~m}^{2}\right)$ for the valve separator with perforation with round holes with a diameter $d_{0}(\mathrm{~m})$, when $\omega_{y}=\pi d_{0}^{2} b_{1} b_{2} z$, where $b_{1}$ is the number of throttle holes in one row; $b_{2}$ is the number of rows.

Expression (5) includes a parameter $M$ as a random component of the angular momentum of the vapor-gas system inside the bubble, the value of which is determined using the calculation method proposed in $[22,24]$ together with the energy parameter $E_{0}$.In this case, the thermodynamic relation is used for the adiabatic process for the vapor-gas system inside the bubble [26], as well as the balance equation between the energy of the bubble system during their stochastic movement and the energy that is spent on the hydrodynamic disruption of the fluid flow over a given period of time [22, 24].

The substitution of expression (3) for the bubble energy during its stochastic movement $E(\lambda, \mu)$ with auxiliary dependences $q_{1}(\lambda), q_{2}(\lambda)$ from (4), (5) into expression (2) leads to the desired expression for the differential function of the distribution of bubbles by their specific sizes according to definition (1).

In addition, the limiting value $K_{v y}^{\max }$ for the conditional valve capacity $K_{v y}\left(\mathrm{~m}^{3} / \mathrm{h}\right)$ was taken into account at its maximum degree of opening $z=1$ according to the simulation results of the differential distribution function of the bubbles with respect to the parameter $\mathrm{z}$ from $[23,24,26]$. Therefore, from (1) - (3) we obtain

$$
\varphi_{1}(\lambda)=\left[\frac{\psi_{1}^{2} E_{0}}{\psi_{2} q_{1}(\lambda)}\right]^{1 / 2} \operatorname{erf}\left(v_{L}\left[\frac{q_{1}(\lambda)}{E_{0}}\right]^{1 / 2}\right) \exp \left[-\frac{q_{2}(\lambda)}{E_{0}}\right],
$$


where, when designating the maximum values of the nominal diameter $D_{y}^{\max }(\mathrm{m})$ and fluid flow through the regulating device $Q_{1}^{\max }\left(\mathrm{m}^{3} / \mathrm{h}\right)$, we have

$$
\begin{aligned}
& \psi_{1}=\left(\frac{k_{0}}{2 \pi}\right)^{2}\left(\frac{D_{y}^{\max }}{D_{y}}\right)^{1 / 4}\left[2 \pi^{4} r_{c}\left(\varepsilon_{1}+\frac{\varepsilon_{2}}{2}+\frac{\varepsilon_{3}}{3}\right)\left(2^{1 / 2}-\frac{1}{3}\right)\left(\frac{K_{v y}^{\max }}{K_{v y}}\right)^{1 / 2}\right]^{-1},
\end{aligned}
$$

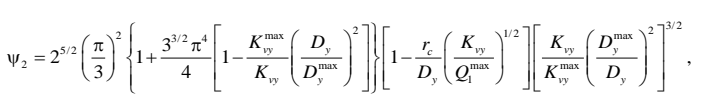

$$
\begin{aligned}
& k_{0}=32 \pi^{1 / 2} /\left(3 D_{y}^{2}\right)-k_{5}, \\
& \varepsilon_{1}=\left(s_{0}-s_{1}\right)\left(c_{0}-c_{1}\right), \\
& \varepsilon_{2}=c_{1}\left(s_{0}-s_{1}\right)+s_{1}\left(c_{0}-c_{1}\right), \\
& \varepsilon_{3}=c_{1} \mathrm{~S}_{1}, \\
& s_{0}=\left[E_{0} / q_{1}(1)\right]^{1 / 2} \operatorname{erf}\left\{v_{L}\left[q_{1}(1) / E_{0}\right]^{1 / 2}\right\} \text {, } \\
& S_{1}=\frac{a_{5} v_{L}^{2}}{a_{4}}\left[\frac{v_{L}}{\pi^{1 / 2}} \exp \left(-\frac{a_{4}^{1 / 2}}{E_{0} r_{c}}\right)-\frac{c_{0}}{2}\right], \\
& c_{0}=\exp \left[-q_{2}(1) / E_{0}\right], \quad c_{1}=c_{0}\left(5 k_{3} M^{2} / r_{c}^{5}-a_{2}\right) / E_{0}, \\
& a_{2}=r_{c}^{2}\left(2 k_{4}+3 k_{5} r_{c}\right), a_{4}=\left(k_{1} r_{c}^{4}+k_{2}\right) v_{L}^{2} \text {, } \\
& a_{5}=3 k_{1} r_{c}^{4}-k_{2} \text {. }
\end{aligned}
$$

In expression (6), the error function $\operatorname{erf}(\gamma)=2 \pi^{-1} \int_{0}^{\gamma} \exp \left(-\theta^{2}\right) d \theta$ with an argument is used $\gamma=2^{-1} \theta^{1 / 2}$.

Obtained in expression (6) of the differential function of the distribution of bubbles by their specific sizes $\varphi_{1}(\lambda)$, taking into account (7) -(13), can be used to calculate the ensemble average characteristics of the radius, diameter and surface for the macroscopic system of bubbles in the region of the valve separator using the expressions

$$
\begin{aligned}
& r_{s b}=r_{c} \int_{u} \lambda d N, \\
& D_{s b}=2 r_{c} \int_{u} \lambda d N, \\
& S_{s b}=4 r_{c}^{2} \int_{u} \lambda^{2} d N .
\end{aligned}
$$

As shown by practical calculations, when performing integration on the right-hand sides of expressions (14), (15) according to (1), (2) for satisfactory convergence with experimental data [26], it is sufficient to take into account linear approximations for the error functions $\operatorname{erf}(\gamma)=2 \pi^{-1 / 2} \gamma+O\left(\gamma^{3}\right)$ in expression (6) and exponential dependence $\exp (-x)=1-x+O\left(x^{2}\right)$ when expanded in a Maclaurin series. Then expressions (14), (15) for the values of the ensemble average characteristics of the diameter and surface for the macroscopic system of bubbles at the early stage of hydrodynamic cavitation in the axial valve separator take the form

$$
\begin{aligned}
& D_{s b}=\frac{r_{c} \psi_{1}}{\psi_{2}^{1 / 2}}\left(\frac{\varepsilon_{1}}{2}+\frac{\varepsilon_{2}}{3}+\frac{\varepsilon_{3}}{4}\right) \varepsilon_{4}, \\
& S_{s b}=\frac{r_{c} \psi_{1}}{\psi_{2}^{1 / 2}}\left(\frac{\varepsilon_{1}}{3}+\frac{\varepsilon_{2}}{4}+\frac{\varepsilon_{3}}{5}\right) \varepsilon_{5},
\end{aligned}
$$

where the coefficients $\psi_{1}, \psi_{2}, \varepsilon_{j}, j=\overline{1,3}$ are given by expressions (7), (8), (10) and correspond to the design parameters of the separators, the operational characteristics of the liquid throttling process, and the physical and mechanical properties of the system; the constants are respectively equal $\varepsilon_{4}=4 \pi^{3}, \varepsilon_{5}=\pi^{5} / 4$.

\section{Results and Discussion}

Set the input parameters of the model. Design parameters for the axial valve separator: hole diameter is $d_{0}=3.5 \times 10^{-3} \mathrm{~m}$; the number of throttle holes in one row is $b_{1}=16$; number of rows is $b_{2}=5$. Performance parameters for the process of throttling the liquid in the separator (for calculating the parameters $M$ and $E_{0}$ according to $[22,24])$ : the maximum achievable liquid flow through the separator is $Q_{1}^{\max }=0.5 \mathrm{~m}^{3} / \mathrm{h}$; minimum pressure drop is $\Delta p_{\text {min }}=1.5 \mathrm{kPa}$; medium temperature is $t_{1}=30^{\circ} \mathrm{C}$; the maximum pressure in the center of the bubble is $p_{\max }=1.3 \times 10^{5} \mathrm{kPa}$, which corresponds to the minimum value of its radius $r_{c}=r_{\min }=10^{-3} \mathrm{~m}$ [27]; pressure in the center of the bubble with an averaged radius $p_{s}=10^{-3} \quad \mathrm{~Pa}$ [27]. Physico-mechanical characteristics: for water (density is $\rho_{L}=10^{3} \mathrm{~kg} / \mathrm{m}^{3}$; surface tension coefficient is $\sigma=7.28 \times 10^{-4} \mathrm{~N} / \mathrm{m}$; bulk density is $\gamma_{0}=0.995 \mathrm{~g} \cdot \mathrm{s} / \mathrm{cm}^{3}$; kinematic viscosity $v_{1}=0,81 \cdot 10^{-2} \mathrm{~cm}^{2} / \mathrm{s}$ at $t_{1}=30^{\circ} \mathrm{C}$ ); for air (density is $\rho_{g}=1.205 \mathrm{~kg} / \mathrm{m}^{3}$; adiabatic index is $k=1.3$ [27];); for steam (density is $\rho_{s}=1.44 \times 10^{-2} \mathrm{~kg} / \mathrm{m}^{3}$ ) with a proportion of the content of steam and gas in the internal cavity is $\alpha_{p}: \alpha_{g}=1: 1$.

Using the specified input data, the values of the ensemble mean diameter and surface characteristics $D_{s b}, S_{s b}$ for the macro-system of bubbles in the separator were calculated according to expressions (17), (18). The obtained numerical values of the integral characteristics for the bubble, depending on the degree of opening of the valve opening $\mathrm{z}$ and conditional values of the Reynolds criterion $\operatorname{Re}_{y}$, are contained in Table 1 . 
Table 1. The results of the calculation of the ensemble mean diameter and surface characteristics for the macro-system of bubbles in the separator

\begin{tabular}{|c|c|c|c|c|}
\hline № & $\mathbf{1}$ & $\mathbf{2}$ & $\mathbf{3}$ & $\mathbf{4}$ \\
\hline$z$ & 0.2 & 0.5 & 0.8 & 1.0 \\
\hline $\mathrm{Re}_{y}, 10^{4}$ & 1.55644 & 0.98438 & 0.77822 & 0.69606 \\
\hline$D_{s b}, 10^{-2}, \mathrm{~m}$ & 0.1610 & 0.1726 & 0.1785 & 0.1646 \\
\hline$S_{s b}, 10^{-6}, \mathrm{~m}$ & 3.0279 & 3.2351 & 3.2921 & $3 / 0291$ \\
\hline
\end{tabular}

In addition, the corresponding graphs were constructed for the dependences of the indicated bubble characteristics on the main indicators of the liquid throttling process in the axial valve separator, shown in Fig. 1, 2. Endpoints of graphs 1-4 in Fig. 1a and Fig. 2a correspond to the nominal diameter $D_{y j}, j=\overline{1,4}$ for various degrees of valve opening $\mathrm{z}$.

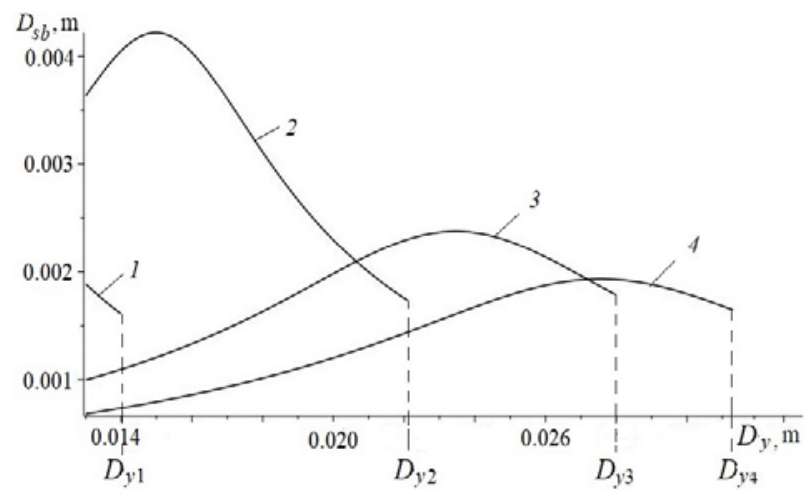

a)

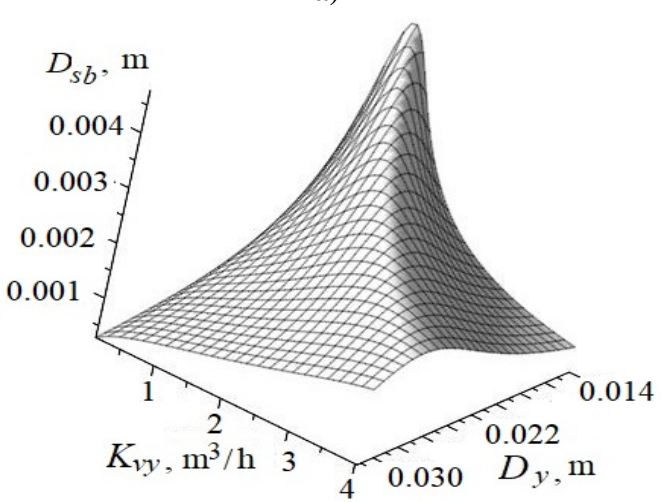

b)

Fig. 1. Dependence of the ensemble average bubble diameter on the main indicators of the liquid throttling process in the axial valve separator: a) $D_{s b}\left(D_{y}\right)$; b) $D_{s b}\left(K_{v y}, D_{y}\right) ; 1-D_{y 1}=$ $1.40^{\times} 10^{-2} \mathrm{~m} ; K_{v y 1}=1.375^{\times} 10^{-1} \mathrm{~m}^{3} / \mathrm{h} ; 2-D_{y 2}=2.21^{\times} 10^{-2} \mathrm{~m} ;$ $K_{v y 2}=1.1891 \mathrm{~m}^{3} / \mathrm{h} ; 3-D_{y 3}=2.80^{\times} 10^{-2} \mathrm{~m} ; K_{v y 3}=2.921 \mathrm{~m}^{3} / \mathrm{h} ; 4$ $-D_{y 4}=3.13^{\times} 10^{-2} \mathrm{~m} ; K_{v y 4}=4.032 \mathrm{~m}^{3} / \mathrm{h}$

It was found that when the valve is fully open ( $z=$ 1.0 ), the ensemble average diameter of the bubble is comparable with this indicator at the initial stage of opening of the throttle holes, when $z=0.2$ (see end points of graphs 1 and 4, Fig. 1a), but less 1.1 times than with $z=0 / 8$ (see endpoints of graphs 3 and 4, Fig. 1a).

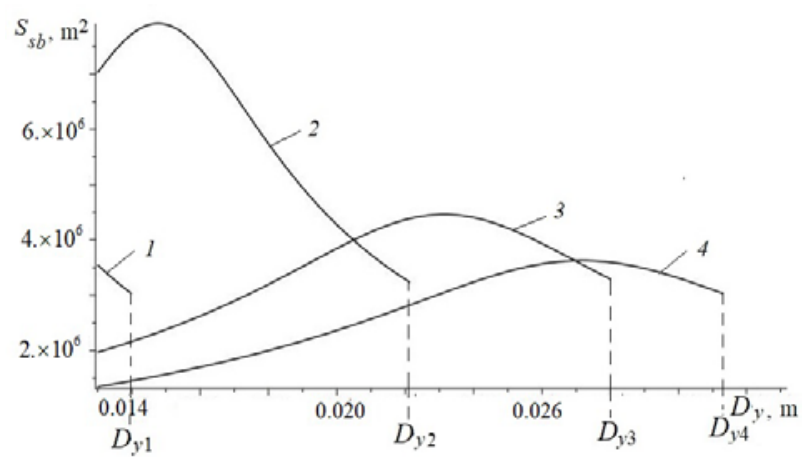

a)

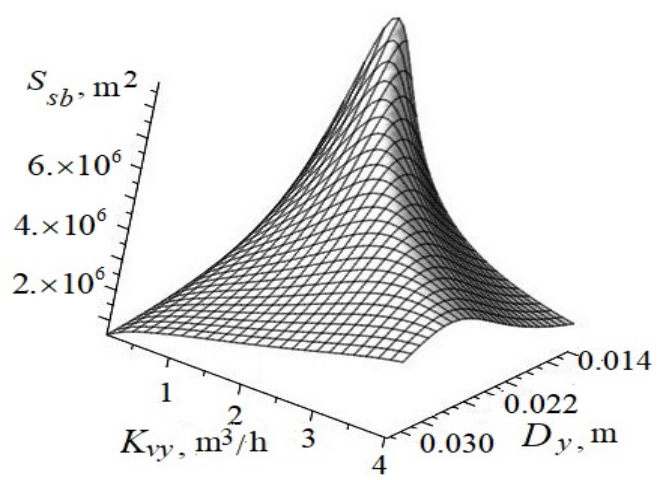

b)

Fig. 2. Dependence of the ensemble average bubble surface area on the main indicators of the liquid throttling process in the axial valve separator: a) $S_{s b}\left(D_{y}\right)$; b) $S_{s b}\left(K_{v y}, D_{y}\right) ; 1-D_{y 1}=$ $1.40^{\times} 10^{-2} \mathrm{~m} ; K_{v y 1}=1.375^{\times} 10^{-1} \mathrm{~m}^{3} / \mathrm{h} ; 2-D_{y 2}=2.21^{\times} 10^{-2} \mathrm{~m}$; $K_{v y 2}=1.1891 \mathrm{~m}^{3} / \mathrm{h} ; 3-D_{y 3}=2.80^{\times} 10^{-2} \mathrm{~m} ; K_{v y 3}=2.921 \mathrm{~m}^{3} / \mathrm{h} ; 4$ $-D_{y 4}=3.13^{\times} 10^{-2} \mathrm{~m} ; K_{v y 4}=4.032 \mathrm{~m}^{3} / \mathrm{h}$

Thus, with an increase in the nominal throughput of the valve $K_{v y}$, when the ratio of its values $K_{v y 3} / K_{v y 1}=2.1$ when opening the separator from $20 \%$ to $80 \%$, the average diameter of the bubbles first increases with the ratio of their values $D_{s b 3} / D_{s b 1}=1.1$ (see end points of graphs 1 and 3, Fig. 1a), and then - a decrease in the characteristic $D_{s b}$ to almost its initial value $D_{s b 1} \approx D_{s b 4}$ with an increase in throughput $K_{v y 4} / K_{v y 3}=1.4$ (see end points of graphs 1 and 4, Fig. 1a). A more detailed description of dependence behavior $D_{s b}\left(K_{v y}, D_{y}\right)$ can be seen in Fig. $1 \mathrm{~b}$ for the corresponding surface.

A similar behavior is observed for the ensemble average bubble area from the main indicators of the throttling process of the fluid flow in the axial valve 
separator (Fig. 2a and Fig. 2b). With an increase in the diameter of the conditional passage section by 2 times, when $D_{y 3} / D_{y 1}=2$ the average bubble area increases with the ratio of values $S_{s b 3} / S_{s b 1}=1.09$ (see end points of graphs 1 and 3, Fig. 2a), and when we go to the maximum value $D_{y 4}$ with the corresponding ratio $D_{y 4} / D_{y 3}=1.2$, the average bubble area decreases to the initial values $S_{s b 1} \approx S_{s b 4}$ (see end points of graphs 1 and 4, Fig. 2a). The behavior of the dependence $S_{s b}\left(K_{v y}, D_{y}\right)$ is traced in Fig. 2b.

\section{Conclusions}

So, when studying the process of fluid throttling in an axial valve, a number of factors have been identified that need to be taken into account when describing the intensity of bubble formation at an early stage of hydrodynamic cavitation. For example, these include factors related to the physicomechanical properties of the working medium (adiabatic effects for the internal cavity of the bubble, interfacial phenomena), and the design features of the separator (number, shape and location of throttle openings) along with operational characteristics of its operation (maximum achievable fluid flow rate, minimum pressure drop, temperature, etc.). The combination of these factors allowed us to build a set of input parameters of the model. Summing up the research, the following main conclusions and results can be given:

1. Based on the stochastic approach [18] within the framework of the accepted assumptions [22-24], the proposed model for the formation of spherical bubbles in the axial valve separator in the process of fluid throttling. Additionally, the limit value of the conditional valve capacity at the maximum degree of opening was taken into account.

2. The indicated model made it possible to obtain a number of analytical dependences characterizing the process of throttling a liquid in an axial valve separator. These dependences include: the differential distribution function of the bubbles according to their specific sizes in the form (6) and the ensemble-averaged characteristics of the system of bubbles formed diameter and surface area.

3. The results of the stochastic model for the differential function of the distribution of bubbles over their specific sizes in the form (6) and for ensembleaveraged bubble sizes (17) are in agreement with the results of the stochastic model of the authors [26], which describes the behavior of the bubble system by the degree of valve opening in comparison with experimental data.

4. The indicated results both for the function $\varphi_{1}(\lambda)$ from expression (6) and for the ensemble average characteristics of the macroscopic system of bubbles in the axial valve separator in the form of expressions (17) and (18) can be used to formulate an engineering methodology for calculating control equipment.

\section{References}

1. W. H. Besant, Hydrostatics and hydrodynamics Cambridge University Press, London, Art. 158, (1859)

2. J. W. S., Baron Rayleigh, Scientific papers 6 1911-1919; Cambridge University Press, 1899-1920, reissued by the publisher, ISBN 978-0-511-70401-7 (2011)

3. M. S. Plesset and R. B. Chapman, J. Fluid Mech., 47(2), 125-141 (1971)

4. H. Abdulmouti, American Journal of Fluid Dynamics, 4(4), 197-240 (2014)

5. G. L. Chahine, Strong interactions bubble/bubble and bubble/flow Kluwer Academic Publishers, Printed in the Netherlands, 195-206 (1994)

6. J. Qian, B. Liu, Z. Jin, H. Zhang and A. Lu, J. Zhejiang Univ. Sci. A., 17, 54 (2016)

7. A. Adamkowski and M. Lewandowski, J. Hydraul. Eng., 141(2), 04014077 (2015)

8. J. R.Valdés, J. M. Rodríguez, R. Monge, et al., Energy Convers. and Manage, 78, 776-786 (2014)

9. S. Chandrasekhar, Rev. Mod. Phys., 15(1), 1-87 (1943)

10. V. Volmer and A. Weber, J. Phys. Chem., 119, 277-301 (1926)

11. J. Frenkel, Kinetic Theory of Liquids. International Series of Monographs on Physics. (Oxford University Press, London, 1946), 332

12. E. Ellas and P. L. Chambre, Int J. Multiphase Flow, 26, 191-206 (2000)

13. T. S. Shin and O. C. Jones, Int. J. Multiphase Flow, 19(6), 943-964 (1993)

14. N. V. Alekseechkin, J. Aerosol Sci., 116(12), (2017)

15. K. J. Vachaparambil and K. E. Einarsrud. J. Electrochem. Soc., 165(10), E504-E512 (2018)

16. R. F. Ganiev and A. Korneev, Doklady Physics, 59(5), 223-225 (2014)

17. U. Iben, A. Makhnov, A. Schmidt, Journal of Physics: Conf. Series, 1038, 012128 (2018)

18. Yu. L. Klimontovich, Turbulentnoe dvizhenie $i$ struktura khaosa: Novyy podkhod k statisticheskoy teorii otkrytykh system [Turbulent motion and chaos structure: A new approach to the statistical theory of open systems], 4rd ed. (Moscow: LENAND, 2014), pp. 328

19. A. B. Kapranova and I. I. Verloka, Theor. Found. of Chem. Eng., 52(6), 1004-1018 (2018)

20. I. Verloka, A. Kapranova, M. Tarshis and S. Cherpitsky, Int. J. Mech. Eng. Technol., 9(2) 438-444 (2018)

21. A. E. Lebedev, A. B. Kapranova, A. M. Melzer, et al., Patent on the model 175776 Russian Federation, IPC F16K 1/12, F16K 47/14, F16K 3/24. Direct-acting control valv, Bulletin of Inventions of the Russian Federation 3 (2018)

22. A. Kapranova, A. Lebedev, A. Melzer and S. Neklyudov, Int. J. Mech. Eng. Technol., 9(3) 25-31 (2018)

23. A. Kapranova, S. Neklyudov, A. Lebedev and A. Melzer, Int. J. Mech. Eng. Technol, 9(8) 153-159 (2018)

24. A. Kapranova, S. Neklyudov, A. Lebedev and A. Melzer, Int. J. Mech. Eng. Technol, 9(8) 160-166 (2018) 
25. A. B. Kapranova, A. E. Lebedev, S. A. Solopov and A. M. Melzer, Czasopismo techniczne. Mechanika. (Krakov, Poland). 113(2), 136-144 (2016)

26. A. Kapranova and A. Miadonye, J. Oil, Gas Petrochem. Sci., 2(2), 70-75 (2019)

27. E. P. Zaporozhets, L. P. Kholpanov, G. K. Siebert and A. V. Artemov, Theor. Found. of Chem. Eng., 38(3), 225-234 (2004) 